Proceedings of the ISCIE International Symposium on Stochastic Systems Theory and Its Applications Osaka, Nov. 10-12, 1993

\title{
Estimation of Fluid Flow Resistance
}

\author{
of Elastic Tube \\ Masanori Sugisaka and Takeshi Nogai \\ Dept. of Electrical and Electronic Engineering \\ Oita University \\ Oita, 870-11, Japan

\begin{abstract}
This paper developed a new device which is able to identify the unknown water flow resistance of elastic tubes from a direct part of the measured water pressures by circulating pulsatile water flow in tubes with a roller pump. The device consists of two pressure sensors, A/D transducer board, one roller pump with constant revolutional speed, a low-pass filter to cut off high frequency noise, and microcomputer with a special software to identify the unknown parameters of the tube. The water flow resistance $\mathrm{R}$ is identified by the on-line nonlinear least squares method.
\end{abstract}

\section{INTRODUCTION}

Recently, we developed a two-step method[1][2] for estimating the unknown physiological parameters of a blood tube in the artery from the measured data of the blood pressure at a certain place on the blood tube, using a distributed parameter electric circuit model shown in Fig.1, namely, the telegraphic equation which is able to describe the hemodynamics of the blood pressure and flow system [3][4]. This method uses two step logic in order to identify the unknown physiological parameters from the measured blood pressure. At the first logic the unknown blood flow resistance $\mathrm{R}$ and blood flow conductance $\mathrm{G}$ are identified from the direct current component of measured blood pressure and at the second logic the unknown blood flow inductance $L$ and blood flow capacitance $C$ are identifled from the alternating current component. It is seemed that the two step logic architecture is intuitively suited for the development of a medical device in order to identify the unknown parameters of artificial blood tubes in a blood circular system.

In this paper developed a new device to identify the water flow resistance $R$ from the direct part of measured water pressure data by the on-line nonlinear least squares method. This paper introduces briefly the system of developed experimental device (identifier) in Section 2 and the methods employed for estimating the water flow resistance in Section 3. Some of experimental results are illustrated in section 4 and conclusion follows in section 5 .

\section{SYSTEM OF IDENTIFIER}

The system of experimental device in order to identify the parameters is illustrated in Fig.2. Specifications for both roller pump named as microtube pump commercially. and pressure transducer are listed in Table 1 . The system of identifier consists of micro-tube pump, two pressure transducers which are located at a certain distance and are connected to A/D transducer board, water tube in order to circulate fluid, microcomputer which implements the calculation of unknown resistance etc., and a tube to be tested.

The water is pumped out from the micro-tube pump and is circulated into the tube to be tested where two pressure transducers are located at inlet and outlet of the tube. The 
pressures of inlet and outlet water are measured by the A/D transducer boad or sensors at each sampling interval. Thereafter the identification of unknown water flow resistance of elastic tube is performed by a microcomputer on making use of the nonlinear least-squares estimation method.

\section{MODEL}

The hemodynamics of blood pressure $v(\mathrm{x}, \mathrm{t})$ and blood flow $1(x, t)$ in arteries in cardiovascular system is approximately modeled [1][5] by the distributed parameter electric circuit shown in Fig.1 described by

$$
\begin{aligned}
& -(\partial / \partial \mathrm{x}) v(\mathrm{x}, \mathrm{t})=\mathrm{R} i(\mathrm{x}, \mathrm{t})+\mathrm{L}(\partial / \partial \mathrm{t}) v(\mathrm{x}, \mathrm{t}), \\
& -(\partial / \partial \mathrm{x}) i(\mathrm{x}, \mathrm{t})=\mathrm{G} v(\mathrm{x}, \mathrm{t})+\mathrm{C}(\partial / \partial \mathrm{t}) v(\mathrm{x}, \mathrm{t}), \\
& 0 \leq \mathrm{x} \leq 1,0 \leq \mathrm{t} \leq \mathrm{T},
\end{aligned}
$$

where $\mathrm{x}$ is the geometrical point in the blood tube, 1 is the length of the blood tube, $t$ is time, $T$ is the period of the pulsatile blood pressure. The parameters R, G, L, and C are unknown blood flow resistance, conductance, inductance, and capasitance to be identified, respectively. The unit employed for the blood pressure and the unknown parameters is the ciculation unit(CU).

Using the N-terms complex Fourler series expansion the solutions $v(x, t)$ and $1(x, t)$ to Eqs.(1)-(2) are described as

$$
\begin{aligned}
& \begin{aligned}
& v(x, t)=u_{01}(x) / 2+\sum_{k=1}^{N}\left\{u_{k 1}(x) \cos \omega k t\right. \\
&+\left.u_{k_{2}}(x) \sin \omega k t\right\}
\end{aligned} \\
& \begin{aligned}
1(x, t)=u_{\theta_{3}}(x) / 2+\sum_{k=1}^{N}\left\{u_{k 3}(x) \cos \omega k t\right. \\
+
\end{aligned} \\
& \left.+u_{k 4}(x) \sin \omega k t\right\}
\end{aligned}
$$

where $u_{k 1}, u_{k_{2}}, u_{k} 3$, and $u_{k} 4$ satisfy the set of ordinary differential euation given by

$$
\left(\begin{array}{l}
\dot{u}_{k 1} \\
\dot{u}_{k 2} \\
\dot{u}_{k 3} \\
\dot{u}_{k 4}
\end{array}\right)=-\left(\begin{array}{cccc}
0 & 0 & R & \omega k L \\
0 & 0 & -\omega k L & R \\
G & \omega k C & 0 & 0 \\
-\omega k C & G & 0 & 0
\end{array}\right)\left(\begin{array}{l}
u_{k 1} \\
u_{k 2} \\
u_{k 3} \\
u_{k 4}
\end{array}\right),
$$

$$
u_{B 2}(x)=u_{B A}(x)=0, k=0,1, \ldots, N \text {. }
$$

We can use the above model in order to describe the fluid dynamics of water which circulates into the experimental device approximately. Then, for this case the word "blood" is relaced by "water". In the following we regard blood used in the above as water.

\section{IDENTIFICATTON LOGIC}

We will show identification logic for the water flow resistance in the followings.

The equation which satisfies the direct part of measured water pressure is obtained by equating $\mathrm{k}=0$ in $\mathrm{Eq} .(6)$, we have

$$
\begin{aligned}
{\left[\begin{array}{l}
\dot{u}_{\theta_{1}}(x) \\
\dot{u}_{\theta_{3}}(x)
\end{array}\right] } & =-\left(\begin{array}{ll}
0 & R \\
G & 0
\end{array}\right)\left[\begin{array}{l}
u_{\theta_{1}}(x) \\
u_{\theta_{3}}(x)
\end{array}\right), \\
& =-\left(\begin{array}{rr}
0 & \theta_{1} \\
\theta_{2} & 0
\end{array}\right)\left[\begin{array}{l}
u_{\theta_{1}}(x) \\
u_{\theta_{3}}(x)
\end{array}\right],
\end{aligned}
$$

where $R=\theta_{1}$ and $G=\theta_{2}$. The inftial conditions for Eqs. (8)-(9) are given by

$$
\left(\begin{array}{ll}
u_{\theta_{1}} & (0) \\
u_{\theta_{3}} & (0)
\end{array}\right)=\left(\begin{array}{l}
C_{1} \\
C_{2}
\end{array}\right)
$$

It is noted that $C_{1}$ is the initial water pressure at a reference point and $C_{2}$ is the initial water flow.

The measured data of water pressure is expressed by

$$
\begin{aligned}
& y\left(x_{i}, k \Delta t\right)=u_{\theta_{1}}\left(x_{i}, k \Delta t\right) / 2+\varepsilon(k \Delta t), \\
& k=0,1, \ldots, N, \\
& 1=0,1, \ldots, M,
\end{aligned}
$$

where $N$ is number of the data, $M$ is number of sensors and $\varepsilon(k \Delta t)$ is the error involved in modelling and calculation or measured noise.

The identification algorithm used in this experiments is the discrete-time on-line nonlinear least-squares algorithm. The measured data is the calculated by direct current of the water pressure, namely, the data based on 
one sensor specified by

$$
\begin{aligned}
& y_{t}=u_{01}(x) / 2(\text { const. })+\varepsilon t \\
& t=1, \ldots, m .
\end{aligned}
$$

Given the measured data $\left\{y_{t}, 0 \leq t \leq m\right\}$ the unknown parameter $\theta=\left[\begin{array}{ll}\theta_{1} & \theta_{2}\end{array}\right]^{\top}=\left[\begin{array}{ll}R & G\end{array}\right]^{\top}$ ( $\mathrm{T}:$ transpose) is identified such that

$$
J=\sum_{t=1}^{M}\left(y_{t}-u_{t_{1}}(x) / 2\right)^{2}
$$

is minimized. The nonlinear least-squares algorithm is given by

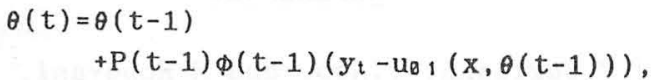

$\theta(0)=0$ (Initial Condition),

$$
\begin{aligned}
P(t-1)= & P(t-2)-P(t-2) \phi(t-1) \phi^{\top}(t-1) P(t-2) \\
& /\left[1+\phi^{\top}(t-1) P(t-2) \phi(t-1)\right],
\end{aligned}
$$

$P(0)=\alpha I(\alpha>>1)$ (Initial Condition),

where $I$ is the unit matrix, $P$ is $2 \times 2$ matrix, $\alpha$ is gain, and $\phi$ is specifled by

$$
\phi(t-1)=\left.\left[d_{\theta},(x, 0) / d \theta\right]\right|_{\theta=8}(t-1)
$$

In the above equation, the sensitivity of the function $u_{0}$ with respect to $\theta$ is calculated by a set of ordinary differential equations:

$$
\begin{aligned}
-d u_{\theta_{1}}(x) / d \theta_{1}= & u_{\theta_{3}}(x) \\
& +\theta_{1}(t-1) d u_{\theta_{3}}(x) / d \theta_{1}, \\
-d u_{\theta_{1}}(x) / d \theta_{2}= & \theta_{1}(t-1) d u_{\theta_{3}}(x) / d \theta_{2}, \\
-d u_{\theta_{3}}(x) / d \theta_{1}= & \theta_{2}(t-1) d u_{\theta_{1}}(x) / d \theta_{1}, \\
-d u_{\theta_{3}}(x) / d \theta_{2}= & u_{\theta_{1}}(x) \\
& +\theta_{2}(t-1) d u_{\theta_{1}}(x) / d \theta_{2},
\end{aligned}
$$

The flowchart of the nonlinear least-squares method is illustrated in Fig.3. We notice that if there is no divergent in the elastic tube then the water flow conductance $\mathrm{G}=\theta_{2}$ is equal to zero. For this case the identification algorithm stated above is simplified such that $\theta$ and $P$ in (14)-(17) become scalar values and only the sensitivity functions duo $(x) / d \theta_{1}$ and $\mathrm{du}_{3}(\mathrm{x}) / \mathrm{d} \theta_{1}$ are necessary. Namely, Eqs. (20) and (22) are not necessary. Therefore, the identification logic become a simple one.

\section{EXPERTMENTAL RESULTS}

If there is no divergent in the elastic tube then the water flow conductance $G=\theta_{2}$ is equal to zero. For this case, we notice that the water flow resistance $R$ is calculated from a simple equation as well as the equations stated in Section 4 . The equation is specified by

$$
R=\frac{u_{01}(0)-u_{01}(1)}{u_{0_{3}}(0) 1} \quad\left[\mathrm{dyn} \cdot \mathrm{cm}^{-5} \cdot \mathrm{sec}\right]
$$

where $u_{b 1}(0)\left(\right.$ dyne $\left./ \mathrm{cm}^{2}\right)$ is the direct part of the water pressure of the pressure transducer 1 and $u_{0}(1)\left(d y n e / \mathrm{cm}^{2}\right)$ is that of the pressure transducer 2 shown in Fig.1. The value $\mathrm{U}_{3}(0)\left(\mathrm{cm}^{3}\right)$ is the initial water flow. The parameter 1 is the length of the elastic tube between two pressure transducers. In the experiments, we used a micro-pump motor and two pressure transducers. The specification of the micro-pump motor and the pressure transducer are illustrated in Table 1.

The internal diameter of the tube to be tested which, consists of silicone, is $4 \mathrm{~mm}$ and the external diameter of the tube is $6 \mathrm{~mm}$. The sampling time employed is 0.01 second and the number of pressure data measured is set to 1024 in order that the FFT works easily. The FFT method is applied for the measured pressure data in order to get the direct component of the water pressure. The length of the elastic tube, in other words, the distance between the pressure transducer 1 at the reference point (at the outlet of the tube) and the pressure transducer 2 at the certain point, is changed as $20(\mathrm{~cm}), 40(\mathrm{~cm}), 60(\mathrm{~cm})$, and $100(\mathrm{~cm})$. 
Figure 4 shows the measured data of water pressures for pressure sensors 1 and 2 . when the length of the elastic tube is $60.0 \mathrm{~cm}$. Figure 5 shows the corresponding spectrum calculated by the FFT using the Hanning window. The initial conditions $C_{1}=u_{0}(0)$ and $\mathrm{C}_{2}=\mathrm{u}_{0_{3}}(0)$ for the nonlinear least-square estimation method given by Eqs (14)-(17) are measured. Since the initial condition $u_{0}(0)$ is the direct part of initial water pressure of the elastic tube at the reference point the pressure is measured from the pressure transducer 1. Since the initial condition $u_{8}(0)$ is the initial water flow, this value is measured by a measuring cylinder such as $\mathrm{U}_{9}{ }_{3}(0)=0.2844\left(\mathrm{~cm}^{3} / \mathrm{sec}\right)$. We note that the theoretical values [4] for the water flow resistance of the elastic tube is calculated by

$$
\mathrm{R}=\frac{8 \mu}{\pi \mathrm{r}_{6}^{4}}
$$

where $\mu$ is the coefficient of viscosity of water and $r_{\theta}$ is the internal radius of the elastic tube. Using the value $\mu=0.01$ (poise) and $\mathrm{r}_{\theta}=0.2(\mathrm{~cm})$ the theoretical value is calculated as follows.

$$
R=15.92\left(\text { dyn } \cdot \mathrm{cm}^{-5} \cdot \mathrm{sec}\right)
$$

In the meanwhile, in order to calculate the water flow resistance by the nonlinear leastsquare estimation method the following initial conditions are used for Eqs.(15) and (17).

$$
\begin{aligned}
& \alpha=10000 \\
& \theta_{1}(0)=0 \quad\left(\theta_{2}(0)\right. \text { is not necessary) }
\end{aligned}
$$

Figure 6 shows the identified water resistances when the tube length is changed as mentioned before.

\section{CONCLUSTONS}

This paper introduced a new device to identify unknown water flow resistance. The two step logic for estimating the unknown parameters of elastic tubes is proposed for the identification architecture which is implemented on a micro computer NEC PC-9801 in the device. It is seen that the logic employed is simple and intuitive. The improvement of the device and noise cut capacitor will be investigated for the practical use in the future.

\section{REFERENCES}

[1] M.Sugisaka, T.Aso, and M.Kobayasi, "Two Step Method of Identification of Physiological Parameters in Arteries," Proc. of Eighth International Conference on Systems Engineering, U.K. pp.810-816, Sept. 10-12,1991.

[2] M.Sugisaka, N.Katsuragi, S.Sagara and R.Fischl, "Parameter Identification of Fluid Tubles,".

Proc. of the IEEE International Conterence on Systems Engineering, Kobe, Japan, pp.381-384 September 17-19, 1992

[3] Z.Nagumo ed., Seitaisisutemu, Sisutemukougakukouza 9 , in Japanese, Nikkankougyoushinbunshya, 1971.

[4] R.Sigiya, M.Mochizuk1, and H.Kanai, Ziyunkankei no Rikigaku to Keisoku, in Japanese, Koronashya, Tokyo, 1971.

[5] S.Oka, Reology-Bioreology-, in Japanese, Syokabo, Tokyo, 1974

[6] M.Sugisaka et al., "A Simulation Study on Identification of Blood Flow Resistance in Arteries," Identification and System Parameter Estimation, pp.1125-1130, 1985. 

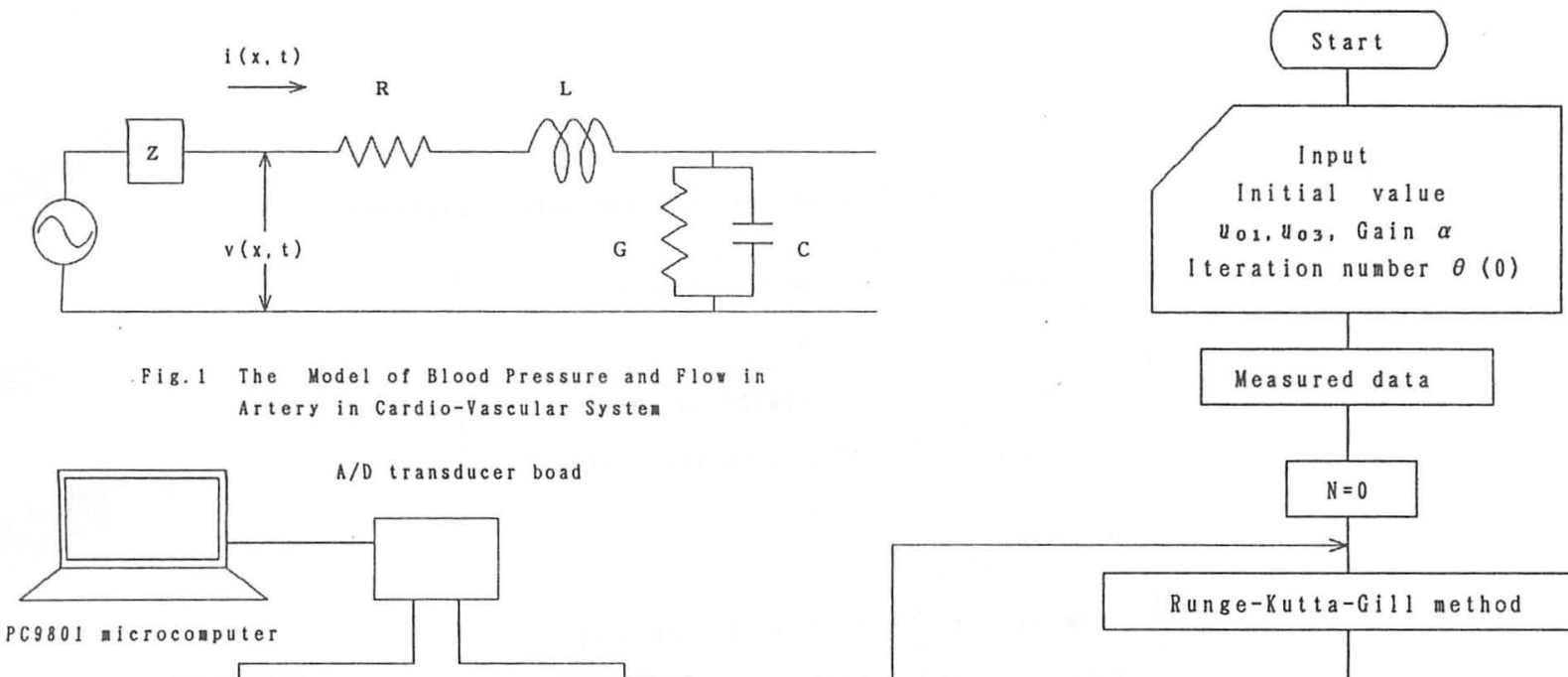

The Model of Blood Pressure and Flow in Artery in Cardio-Vascular Systew

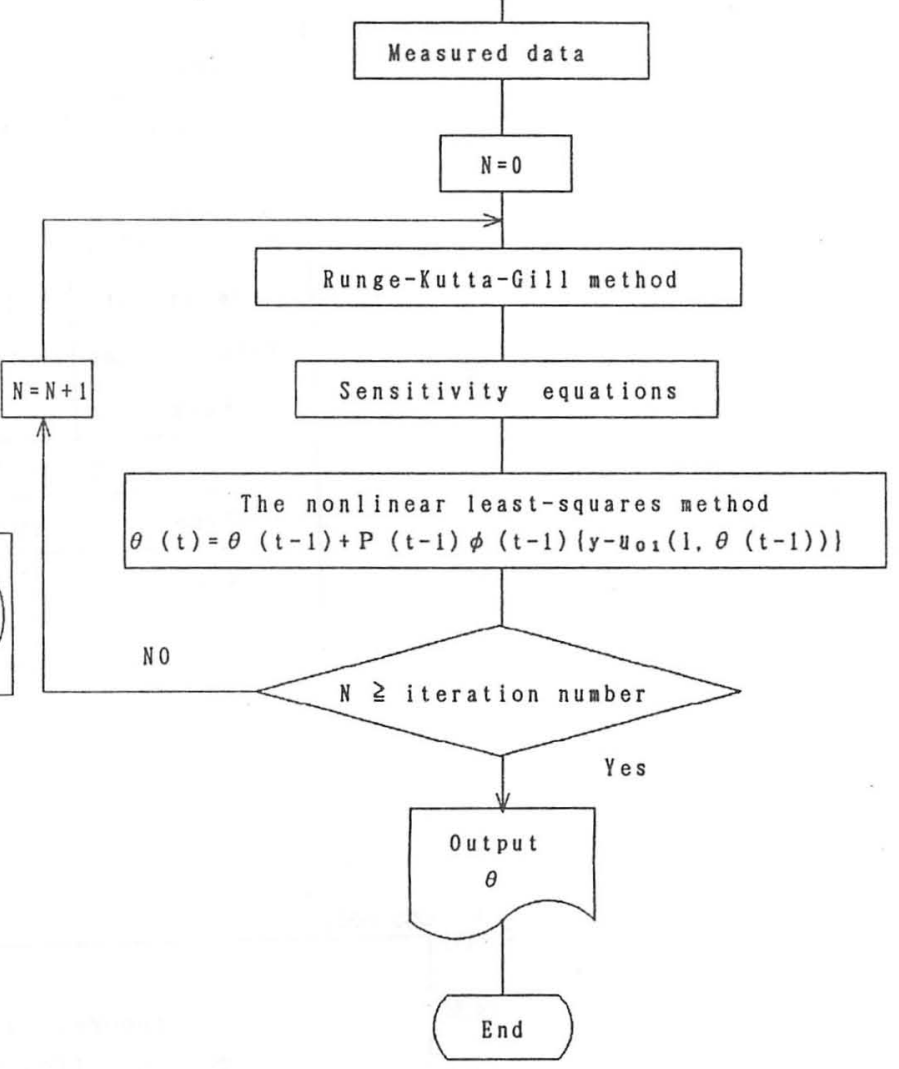

Fig. 3 Flowchart of Nonlinear Least-Squares Method
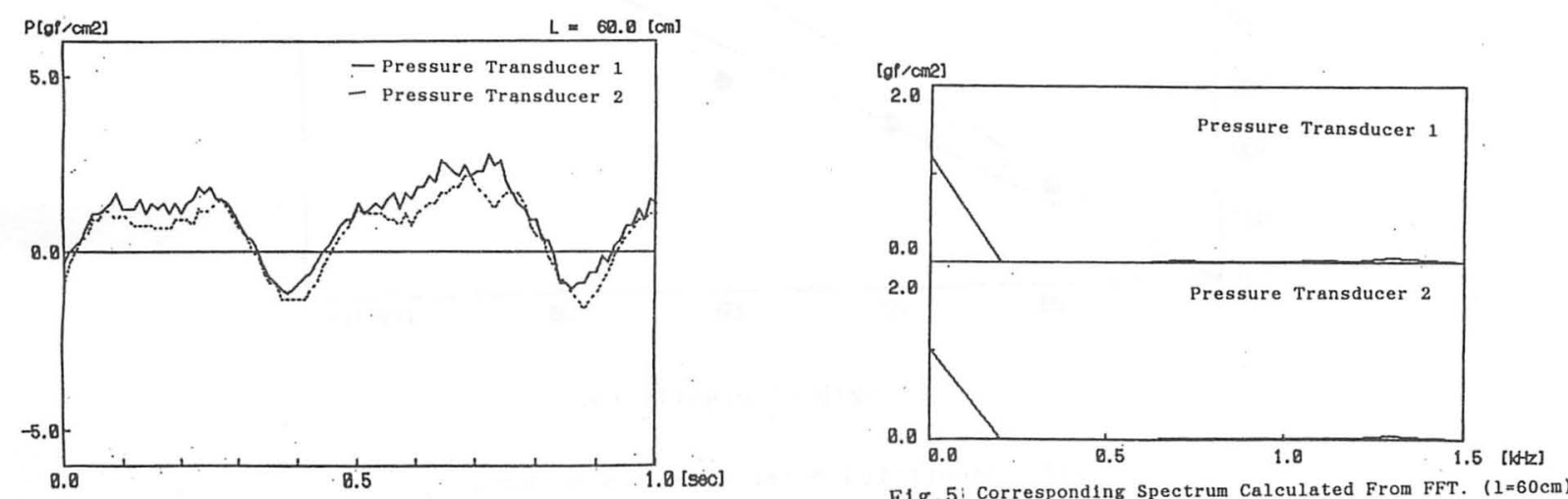

F1g. 4 Measured Data of Water Pressure for Pressure Transducers 1 and 2 of Elastic Tube with $1-60 \mathrm{~cm}$ 
Table 1: Specication of Puap and Pressure Transducer

\begin{tabular}{|c|c|}
\hline It em & Specification \\
\hline \multicolumn{2}{|r|}{$\mathrm{P} u \mathbb{\mathrm { p }}$} \\
\hline Type & TOKYORIKA CO. MP-3 \\
\hline Motor & $\begin{array}{l}\text { AC100V, } 50 / 60 \mathrm{~Hz}, 15 \mathrm{~W} \\
\text { Induction, Speed Variable }\end{array}$ \\
\hline No. of Tubes & 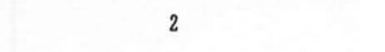 \\
\hline Size of Tube & $1.15 \times 3 \Phi \sim 4 \Phi \times 6 \Phi$ \\
\hline Size of Pump & $150 \mathrm{~W} \times 157 \mathrm{D} \times 185 \mathrm{H}$ \\
\hline Weight & 2. $9 \mathrm{Kg}$ \\
\hline \multicolumn{2}{|c|}{ Pressure Transducer } \\
\hline Type & COPAL CO. PA-500-501G \\
\hline Zero & $1.00 \mathrm{~V}$ \\
\hline Span & $3.99 \mathrm{~V}$ \\
\hline
\end{tabular}

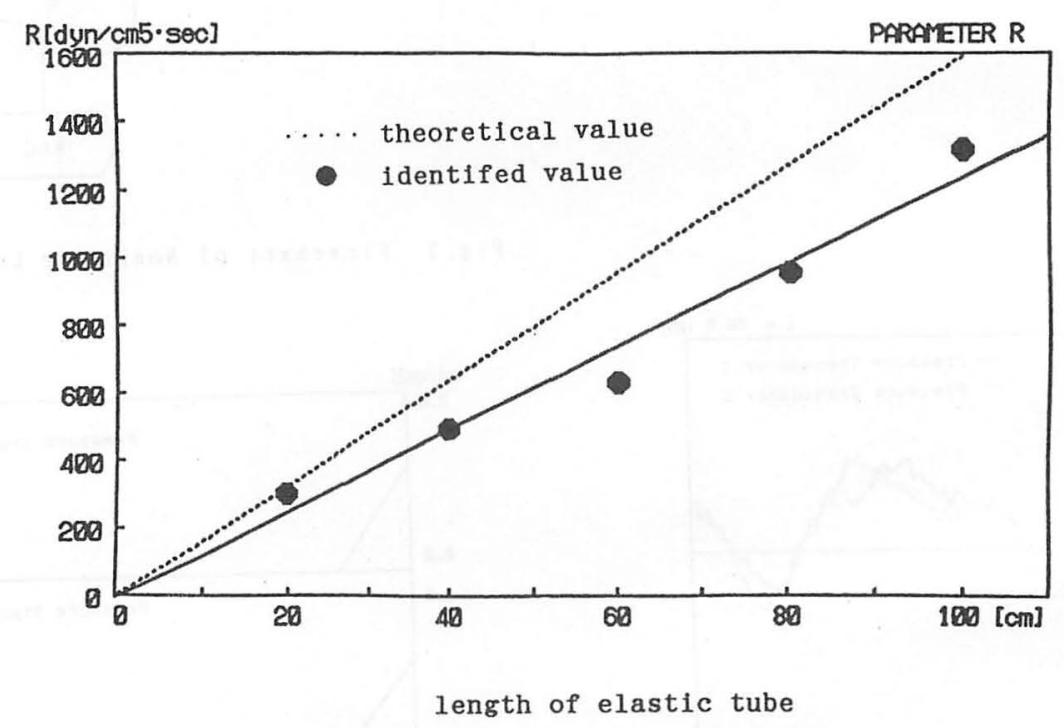

F1g.6 Identified Water Flow Resistances $R$ 